TRANSACTIONS OF THE

AMERICAN MATHEMATICAL SOCIETY

Volume 350, Number 10, October 1998, Pages 3903-3922

S $0002-9947(98) 02008-\mathrm{X}$

\title{
STABILITY RESULTS ON INTERPOLATION SCALES OF QUASI-BANACH SPACES AND APPLICATIONS
}

\author{
NIGEL KALTON AND MARIUS MITREA
}

\begin{abstract}
We investigate the stability of Fredholm properties on interpolation scales of quasi-Banach spaces. This analysis is motivated by problems arising in PDE's and several applications are presented.
\end{abstract}

\section{INTRODUCTION}

In this paper we initiate the study of stability of Fredholm properties of operators on complex interpolation scales of quasi-Banach spaces. As such, this is a natural continuation and extension of previous work in the literature (cf., e.g., the articles [32], [35], [7], [8]) which only deals with the case of Banach spaces.

The first task is to identify the essential functional analytic elements such that a satisfactory stability theory can be developed in the context of quasi-Banach spaces. One of the main problems is that we are forced to work with non-locally convex spaces and, consequently, several central theorems from the Banach space setting are no longer available (most notably duality results based on the HahnBanach theorem). Let us point out that all existing papers addressing similar issues employ in one form or another results from Banach space theory which do not carry over to the quasi-Banach context (for instance, it is assumed that the dual of the interpolation scale is an interpolation scale itself). Thus, it is desirable to devise a different approach which, in particular, avoids the use of duality.

In section 2 we indicate that this can be accomplished in a rather general, flexible framework. For instance, here we show that for an interpolation operator being an isomorphic embedding or being onto are stable properties on interpolation scales of quasi-Banach spaces (cf. Theorem 2.3, Theorem 2.5). The same applies to the property of being Fredholm and to the index of the operator (see Theorem 2.9). In fact the same results can be extended to analytic families of operators (compare with [11]). Let us also note that our approach is constructive in the sense that all perturbation constants explicitly depend on the original ones (this is relevant in a number of applications).

Section 3 contains a discussion of the complex method applied to interpolation couples of quasi-Banach spaces. Among other things, here we examine the role played by the Maximum Modulus Principle in the context of analytic functions with values in quasi-Banach spaces.

Our primary motivation for studying scales of quasi-Banach spaces stems out from certain PDE's problems which involve the family of (real variable) $H^{p}$ spaces

Received by the editors June 10, 1996.

1991 Mathematics Subject Classification. Primary 46A16, 42B20; Secondary 47A53, 46B70.

(C)1998 American Mathematical Society 
for $p \leq 1$. The point is that many perturbations of, say, $H^{1}$-invertibility results are of considerable interest.

In order to make this idea more transparent, recall first an old result due to S. Zaremba ([36]) to the effect that the unique solution of the Dirichlet problem for the Laplacian in Lipschitz domains with Hölder continuous data extends to a Hölder continuous function on the closure of the domain (with the same, small exponent). The original proof is based on the construction of barriers and now the question arises whether it is possible to represent the solution in the layer potential form with a Hölder continuous boundary density (much in the spirit of the $L^{2}$ theory in [34]).

In proving this, a key step is to show that $\frac{1}{2} I+\mathcal{K}^{*}$, the adjoint of the double layer potential operator, is an isomorphism of $H^{p}$ for $1-\epsilon<p<1$ for some small $\epsilon>0$. This has been first proved by R. Brown [4] (at least for starlike Lipschitz domains) by a careful analysis of the Neumann and Regularity problems for the Laplacian with data in $H^{p}, 1-\epsilon<p<1$. Nonetheless, it is natural to investigate if this result can be obtained more directly by combining a theorem of B. Dahlberg and C. Kenig ([14]) concerning the invertibility of $\frac{1}{2} I+\mathcal{K}^{*}$ on $H^{1}$ with a general stability result on the scale $\left\{H^{p}\right\}_{0<p<\infty}$. In fact, this particular question has also been posed to us by Eugene Fabes. To this, we give a positive answer in the final section of the paper.

Our method of proof is quite flexible and here we also show that similar considerations apply to other situations as well. In particular, this is the case for the three dimensional system of elastostatics in Lipschitz domains, for which we are able to further refine the theory developed in [15], [16].

\section{ACKNOWLEDGEMENTS}

The second named author would like to thank Eugene Fabes for several discussions and his interest in this work.

\section{Interpolation SCALES of QUaSi-Banach SPACES. The abstraCt SETUP}

Throughout the paper, for a quasi-normed space $\left(X,\|\cdot\|_{X}\right)$, we shall denote by $\rho=\rho(X)$ its modulus of concavity, i.e. the smallest positive constant for which

$$
\|x+y\|_{X} \leq \rho\left(\|x\|_{X}+\|y\|_{X}\right), \quad x, y \in X
$$

(note that always $\rho(X) \geq 1$ ). We recall the Aoki-Rolewicz theorem [26],[31], which asserts that $X$ can be given an equivalent $r$-norm (where $2^{1 / r-1}=\rho$ ) i.e. a quasinorm which also satisfies the inequality:

$$
\|x+y\| \leq\left(\|x\|^{r}+\|y\|^{r}\right)^{1 / r} .
$$

In general a quasi-norm need not be continuous but an $r$-norm is continuous. We shall assume however, throughout the paper that all quasi-norms considered are continuous: in fact, of course it would suffice to consider an $r$-norm for suitable $r$.

Let $U$ be a fixed, (Hausdorff) locally compact, locally connected topological space, referred to in the sequel as the space of parameters, and let $\delta: U \times U \rightarrow \mathbf{C}$ be a (fixed) continuous function such that $\delta(z, w)=0$ if and only if $z=w$. Also, suppose that $Z$ is a complex (Hausdorff) topological vector space, called the ambient space.

A family $\mathcal{F}$ of functions which map $U$ into $Z$ is called admissible (relative to $U$, $\delta$ and $Z$ ) provided the following axioms are satisfied: 
1. $\mathcal{F}$ is a (complex) vector space endowed with a quasi-norm $\|\cdot\|_{\mathcal{F}}$ with respect to which this is complete (i.e. $\mathcal{F}$ is a quasi-Banach space);

2. the point-evaluation mappings $\operatorname{ev}_{w}: \mathcal{F} \rightarrow Z, w \in U$, defined by $\operatorname{ev}_{w}(f):=$ $f(w)$ are continuous;

3. for any $K$ compact subset of $U$ there exists a positive constant $D_{K}(\mathcal{F})$ such that for any $w \in K$ and any $f \in \mathcal{F}$ with $f(w)=0$, it then follows that the mapping $U \backslash\{w\} \ni z \mapsto f(z) / \delta(z, w) \in Z$ extends to an element in $\mathcal{F}$ and

$$
\left\|\frac{f(z)}{\delta(z, w)}\right\|_{\mathcal{F}} \leq D_{K}(\mathcal{F})\|f\|_{\mathcal{F}}
$$

We remark that, occasionally, we will write $D_{K}$ for $D_{K}(\mathcal{F})$, and $D_{w}$ for $D_{\{w\}}$.

Assume that such an admissible family $\mathcal{F}$ has been fixed (we shall also call the elements of $\mathcal{F}$ admissible functions). Introduce the intermediate spaces $X_{w}, w \in U$, by

$$
X_{w}:=\{x \in Z ; x=f(w) \text { for some } f \in \mathcal{F}\} \subseteq Z .
$$

Under these hypotheses it is trivial to establish:

Lemma 2.1. For each $w \in U$, the intermediate space $X_{w}$ becomes a quasi-Banach space when equipped with $\|x\|_{w}:=\inf \left\{\|f\|_{\mathcal{F}} ; f \in \mathcal{F}, f(w)=x\right\}, x \in X_{w}$.

Furthermore, the modulus of concavity of the interpolation quasi-norm $\|\cdot\|_{w}$ does not exceed $\rho(\mathcal{F})$, and the inclusion $X_{w} \hookrightarrow Z$ is continuous.

Proof. We need only remark that $X_{w}$ is naturally identified with the quotient space $\mathcal{F} / \operatorname{Ker}\left(e v_{w}\right)$.

The collection of all intermediate spaces $\left\{X_{w}\right\}_{w \in U}$ is called an interpolation scale (relative to the space of parameters $U$, the function $\delta$, the ambient space $Z$, and the admissible family $\mathcal{F}$; however, occasionally we may refer to $(U, \delta, Z, \mathcal{F})$ as being an interpolation scale).

Consider next two interpolation scales: $\left\{X_{w}\right\}_{w \in U}$, relative to some ambient space $Z$ and some admissible family $\mathcal{F}$, and $\left\{Y_{w}\right\}_{w \in U}$, relative to another ambient space $W$ and some admissible family $\mathcal{G}$ (note that $U$ and $\delta$ are assumed to be the same for both scales). A family of operators $\left\{T_{w}\right\}_{w \in U}$,

$$
T_{w}: X_{w} \rightarrow Z, \quad w \in U,
$$

will be called an interpolating family of operators, provided the application

$$
\mathcal{F} \ni f \mapsto\left[U \ni w \mapsto T_{w}(f(w))\right] \in \mathcal{G}
$$

is well-defined, linear and satisfies

$$
\|T .(f(\cdot))\|_{\mathcal{G}} \leq M\|f\|_{\mathcal{F}}, \quad f \in \mathcal{F},
$$

for some positive, finite constant $M$. The best constant in (2.3) is called the interpolation norm of $\left\{T_{w}\right\}_{w \in U}$.

Note that, if a single operator

$$
T: \bigcup_{w \in U} X_{w} \rightarrow \bigcup_{w \in U} Y_{w}
$$

maps admissible functions into admissible functions linearly and boundedly, then the "constant" family $T_{w}:=\left.T\right|_{X_{w}}, w \in U$, becomes an interpolation family of analytic operators. In the sequel, we shall call such a mapping $T$ an interpolation operator. 
Lemma 2.2. Assume that $\left\{T_{w}\right\}_{w \in U}$ is an interpolating family of operators between two interpolation scales $\left\{X_{w}\right\}_{w \in U}$ and $\left\{Y_{w}\right\}_{w \in U}$ as before. Then, for each $w \in U$, the operator $T_{w}$ maps the intermediate space $X_{w}$ linearly and boundedly into $Y_{w}$, with norm not exceeding the interpolation norm of the family $\left\{T_{w}\right\}_{w \in U}$.

Proof. The argument is straightforward. Let $M$ estimate the interpolation norm of $\left\{T_{w}\right\}_{w}$ and, for $x \in X_{w}, \epsilon>0$, pick $f \in \mathcal{F}$ such that $f(w)=x$ and $\|f\|_{\mathcal{F}} \leq\|x\|_{w}+\epsilon$. Then, since $T .(f(\cdot)) \in \mathcal{G}$, it follows that $T_{w} x \in Y_{w}$ and

$$
\left\|T_{w} x\right\|_{w} \leq\|T .(f(\cdot))\|_{\mathcal{G}} \leq M\|f\|_{\mathcal{F}} \leq M\left(\|x\|_{w}+\epsilon\right) .
$$

The conclusion follows.

To state the main results of this section, we still need to introduce some more notation. Consider $\left(X,\|\cdot\|_{X}\right),\left(Y,\|\cdot\|_{Y}\right)$ two quasi-Banach spaces and let $T: X \rightarrow Y$ be a linear, bounded operator. Define $\kappa(T ; X, Y)$ to be the smallest constant so that if $y \in Y$, then there exists $x \in X$ so that $T x=y$ and $\|x\|_{X} \leq \kappa(T ; X, Y)\|y\|_{Y}$. Note that, by the Open Mapping Theorem (which remains valid in the context of quasi-Banach spaces; cf. Theorem 1.4 in [26]), $\kappa(T ; X, Y)$ is finite if and only if $T$ maps $X$ onto $Y$.

We also let $\eta(T ; X, Y)$ be the largest constant so that $\eta(T ; X, Y)\|x\|_{X} \leq\|T x\|_{Y}$ for each $x \in X$. Once again by virtue of the Open Mapping Theorem, $\eta(T ; X, Y)$ is strictly positive if and only if the operator $T$ is injective and has closed range.

Finally, if $\left\{T_{w}\right\}_{w \in U}$ is an interpolating analytic family of operators acting between two interpolation scales $\left\{X_{w}\right\}_{w \in U}$ and $\left\{Y_{w}\right\}_{w \in U}$ then, for each $w \in U$, we abbreviate $\kappa\left(T_{w} ; X_{w}, Y_{w}\right)$ and $\eta\left(T_{w} ; X_{w}, Y_{w}\right)$ by $\kappa\left(T_{w}\right)$ and by $\eta\left(T_{w}\right)$, respectively.

Next we can state our first stability result, concerning the ontoness of an interpolation operator.

Theorem 2.3. Assume that $\left\{T_{w}\right\}_{w \in U}$ is an interpolation analytic family of operators between two interpolation scales $\left\{X_{w}\right\}_{w \in U}$ and $\left\{Y_{w}\right\}_{w \in U}$. Furthermore, assume that $\kappa\left(T_{w_{0}}\right)$ is finite for some $w_{0} \in U$. Then there exist a neighborhood $\omega$ of $w_{0}$ in $U$ and a finite positive constant $C$ such that $\kappa\left(T_{z}\right) \leq C$ for each $z \in \omega$.

In order to prove this theorem we shall need a lemma, which is in fact a part of the standard proof of the Open Mapping Theorem.

Lemma 2.4. Suppose that $\left(X,\|\cdot\|_{X}\right),\left(Y,\|\cdot\|_{Y}\right)$ are two quasi-normed spaces such that $X$ is complete. Also, suppose that $T: X \rightarrow Y$ is a linear, bounded operator for which the following property is true: there exist $0<C_{0}<+\infty$ and $0<a<1$ such that for each $y$ in the unit sphere of $Y$ one can find $x \in X$ with $\|x\|_{X} \leq C_{0}$ and $\|y-T x\|_{Y} \leq a$.

Then $T$ is onto and $\kappa(T ; X, Y) \leq C_{1}$ for some $C_{1}$ depending exclusively on $C_{0}$, $\rho(X)$ and $a$.

Proof. For fixed $y \in Y$, it one can inductively define $\left(x_{n}\right)_{n=1}^{\infty}$ in $X$ so that $\left\|y-\sum_{k=1}^{n} T x_{k}\right\|_{Y} \leq a^{n}\|y\|_{Y}$ and $\left\|x_{n}\right\|_{X} \leq C_{0} a^{n}\|y\|_{Y}$. Then if $2^{1 / r-1}=\rho(X)$ we observe that $\left(\sum_{n=1}^{\infty}\left\|x_{n}\right\|_{X}^{r}\right)^{1 / r} \leq C_{0}\left(1-a^{r}\right)^{-1 / r}\|y\|_{Y}$. Hence $\sum_{n=1}^{\infty} x_{n}=x$ converges and $\|x\|_{X} \leq C_{1}\|y\|_{Y}$ where $C_{1}$ depends on $C_{0}, \rho(X)$ and $a$. Clearly $T x=y$.

We are now in a position to present the proof of Theorem 2.3. 
Proof of Theorem 2.3. Let $\mathcal{F}, \mathcal{G}$ be the two classes of admissible functions corresponding to the two interpolation scales $\left\{X_{w}\right\}_{w \in U}$ and $\left\{Y_{w}\right\}_{w \in U}$. Also, denote by $\rho(\mathcal{F}), \rho(\mathcal{G})$, respectively, their moduli of concavity, and by $M$ the interpolation norm of the family $\left\{T_{w}\right\}_{w}$.

Now, choose a neighborhood $\omega \subseteq U$ of $w_{0}$ such that $\left|\delta\left(z, w_{0}\right)\right|$ is sufficiently small, uniformly for $z \in \omega$ (this is made precise shortly), and let $y$ be an arbitrary vector in $Y_{z}$ with $\|y\|_{z}=1$. Pick $g \in \mathcal{G}$ such that $g(z)=y,\|g\|_{\mathcal{G}} \leq 2$, and set $\tilde{y}:=g\left(w_{0}\right) \in Y_{w_{0}}$. Note that $\|\tilde{y}\|_{w_{0}} \leq\|g\|_{\mathcal{G}} \leq 2$. Then, by hypothesis, there exists $\tilde{x} \in X_{w_{0}}$ such that $T_{w_{0}} \tilde{x}=\tilde{y}$ and $\|\tilde{x}\|_{w_{0}} \leq \kappa\left(T_{w_{0}}\right)\|\tilde{y}\|_{w_{0}}$. Thus, $\|\tilde{x}\|_{w_{0}} \leq 2 \kappa\left(T_{w_{0}}\right)$ and, therefore, there exists $f \in \mathcal{F}$ so that $f\left(w_{0}\right)=\tilde{x}$ and $\|f\|_{\mathcal{F}} \leq 2 \kappa\left(T_{w_{0}}\right)+1$.

Define now $h: U \rightarrow W$ (recall that $W$ is the ambient space for the interpolation scale $\left.\left\{Y_{w}\right\}_{w \in U}\right)$ by

$$
h(w):=\frac{T_{w} f(w)-g(w)}{\delta\left(w, w_{0}\right)} .
$$

By axiom (3), $h$ belongs to $\mathcal{G}$ and

$$
\begin{aligned}
\|h\|_{\mathcal{G}} & \leq D_{w_{0}} \rho(\mathcal{G})\left(\|T .(f(\cdot))\|_{\mathcal{G}}+\|g\|_{\mathcal{G}}\right) \leq D_{w_{0}} \rho(\mathcal{G})\left(M\|f\|_{\mathcal{F}}+\|g\|_{\mathcal{G}}\right) \\
& \leq D_{w_{0}} \rho(\mathcal{G})\left(2 M \kappa\left(T_{w_{0}}\right)+M+2\right)=: C\left(M, w_{0}, \rho(\mathcal{G}), \kappa\left(T_{w_{0}}\right)\right)<+\infty .
\end{aligned}
$$

The idea is now that, if $x:=f(z) \in X_{z}$, then $\|x\|_{z} \leq\|f\|_{\mathcal{F}} \leq 2 \kappa\left(T_{w_{0}}\right)+1$ and

$$
\begin{aligned}
\left\|T_{z} x-y\right\|_{z} & \leq\left|\delta\left(z, w_{0}\right)\right|\|h(z)\|_{z} \leq\left|\delta\left(z, w_{0}\right)\right|\|h\|_{\mathcal{G}} \\
& \leq\left|\delta\left(z, w_{0}\right)\right| C\left(M, w_{0}, \rho(\mathcal{G}), \kappa\left(T_{w_{0}}\right)\right) .
\end{aligned}
$$

Consequently, if $\omega$ is chosen so that for each $z \in \omega$ one has

$$
\left|\delta\left(z, w_{0}\right)\right| C\left(M, w_{0}, \rho(\mathcal{G}), \kappa\left(T_{w_{0}}\right)\right) \leq \frac{1}{2},
$$

Lemma 2.4 can be used to conclude the proof of the theorem.

Our next result deals with the stability of the property of being an isomorphic embedding for an interpolation operator.

Theorem 2.5. Let $\left\{T_{w}\right\}_{w \in U}$ be an interpolating family of operators between two interpolation scales $\left\{X_{w}\right\}_{w \in U}$ and $\left\{Y_{w}\right\}_{w \in U}$, and assume that $\eta\left(T_{w_{0}}\right)$ is strictly positive at some point $w_{0} \in U$. Then there exist a neighborhood $\omega$ of $w_{0}$ in $U$ and a constant $C$ such that $0<C \leq \eta\left(T_{z}\right)$ for each $z \in \omega$.

First we shall need a technical lemma, essentially to the effect that if an admissible function is large at a certain point in the space of parameters, then it remains large when evaluated at neighboring points.

Lemma 2.6. Consider $\left\{X_{w}\right\}_{w \in U}$ an interpolation scale (relative to some $\delta, Z, \mathcal{F}$ ). Let $K$ be a compact subset of $U$ and suppose $w_{0} \in U$. Then for any $f \in \mathcal{F}$ and $z_{0} \in K$ such that $\rho(\mathcal{F}) D_{K}(\mathcal{F})\left|\delta\left(w_{0}, z_{0}\right)\right| \leq 1$ we have

$$
\left\|f\left(z_{0}\right)\right\|_{z_{0}} \geq \frac{1}{3 \rho(\mathcal{F})}\left\|f\left(w_{0}\right)\right\|_{w_{0}}-\left|\delta\left(w_{0}, z_{0}\right)\right| \frac{\rho(\mathcal{F})}{3} D_{K}(\mathcal{F})\|f\|_{\mathcal{F}} .
$$

Proof. First pick any $g \in \mathcal{F}$ so that $\|g\|_{\mathcal{F}} \leq 2\left\|f\left(z_{0}\right)\right\|_{z_{0}}$ and $g\left(z_{0}\right)=f\left(z_{0}\right)$. Then we may find $h \in \mathcal{F}$ so that $\delta\left(z, z_{0}\right) h(z)=f(z)-g(z)$ and $\|h\|_{\mathcal{F}} \leq D_{K}\|f-g\|_{\mathcal{F}} \leq$ $D_{K} \rho\left(\|f\|_{\mathcal{F}}+2\left\|f\left(z_{0}\right)\right\|_{z_{0}}\right)$ where $\rho=\rho(\mathcal{F})$. It follows that

$$
\left\|f\left(w_{0}\right)-g\left(w_{0}\right)\right\|_{w_{0}} \leq\left|\delta\left(w_{0}, z_{0}\right)\right|\|h\|_{\mathcal{F}}
$$


and, hence,

$$
\left\|g\left(w_{0}\right)\right\|_{w_{0}} \geq \rho^{-1}\left\|f\left(w_{0}\right)\right\|_{w_{0}}-\left|\delta\left(w_{0}, z_{0}\right)\right|\|h\|_{\mathcal{F}} .
$$

Thus,

$$
2\left\|f\left(z_{0}\right)\right\|_{z_{0}} \geq \rho^{-1}\left\|f\left(w_{0}\right)\right\|_{w_{0}}-\left|\delta\left(w_{0}, z_{0}\right)\right| D_{K} \rho\left(\|f\|_{\mathcal{F}}+2\left\|f\left(z_{0}\right)\right\|_{z_{0}}\right) .
$$

Hence, if $D_{K} \rho\left|\delta\left(w_{0}, z_{0}\right)\right| \leq 1$ we have

$$
\left\|f\left(z_{0}\right)\right\|_{z_{0}} \geq \frac{1}{3 \rho}\left\|f\left(w_{0}\right)\right\|_{w_{0}}-\left|\delta\left(w_{0}, z_{0}\right)\right| \frac{D_{K} \rho}{3}\|f\|_{\mathcal{F}}
$$

This completes the proof of the lemma.

Next we are ready to present the proof of Theorem 2.5.

Proof of Theorem 2.5. Let $K$ be a fixed compact neighborhood of $w_{0}$. Suppose $z_{0} \in K$ is such that $D_{K}(\mathcal{G}) \rho(\mathcal{G})\left|\delta\left(w_{0}, z_{0}\right)\right| \leq 1$. Now pick $x \in X_{z_{0}}$ with $\|x\|_{z_{0}}=1$. We can pick $f \in \mathcal{F}$ so that $\|f\|_{\mathcal{F}} \leq 2$ and $f\left(z_{0}\right)=x$. If we set $g(z):=T_{z}(f(z))$ then $g \in \mathcal{G}$ and, if $M$ stands for the interpolation norm of the family $\left\{T_{w}\right\}_{w}$, then $\|g\|_{\mathcal{G}} \leq 2 M$. Hence, by Lemma 2.6,

$$
\left\|T_{z_{0}} x\right\|_{z_{0}} \geq \frac{1}{3 \rho(\mathcal{G})}\left\|T_{w_{0}}\left(f\left(w_{0}\right)\right)\right\|_{w_{0}}-2 M \frac{D_{K}(\mathcal{G}) \rho(\mathcal{G})}{3}\left|\delta\left(w_{0}, z_{0}\right)\right| .
$$

It remains to estimate $\left\|T_{w_{0}} f\left(w_{0}\right)\right\|_{w_{0}}$. We have (again by Lemma 2.6)

$$
\begin{aligned}
\left\|T_{w_{0}} f\left(w_{0}\right)\right\|_{w_{0}} & \geq \eta\left(T_{w_{0}}\right)\left\|f\left(w_{0}\right)\right\|_{w_{0}} \\
& \geq \eta\left(T_{w_{0}}\right)\left(\frac{1}{3 \rho(\mathcal{F})}-\left|\delta\left(z_{0}, w_{0}\right)\right| \frac{D_{K}(\mathcal{F}) \rho(\mathcal{F})}{3}\right) .
\end{aligned}
$$

Substituting back it is clear that we find a neighborhood $\omega$ or $w_{0}$ so that $\eta\left(T_{z_{0}}\right)>$ $\frac{1}{10}(\rho(\mathcal{F}) \rho(\mathcal{G}))^{-1} \eta\left(T_{w_{0}}\right)$ for $z_{0} \in \omega$.

Remark. Essentially the same proof above shows that operator inequalities of the form $\|x\|_{w} \leq C^{\prime}\left\|S_{w} x\right\|_{w} \leq C^{\prime \prime}\left\|T_{w} x\right\|_{w}, x \in X_{w}$, for $\left\{S_{w}\right\}_{w},\left\{T_{w}\right\}_{w}$ two interpolating families of operators on interpolation scales of quasi-Banach spaces are stable (in the natural, obvious sense). In fact, if $\left\{S_{w}\right\}_{w},\left\{T_{w}\right\}_{w}$ are two interpolating families of operators on interpolation scales of quasi-Banach spaces such that, for some $w_{0},\left\|S_{w_{0}} x\right\|_{w_{0}} \leq C\left\|T_{w_{0}} x\right\|_{w_{0}}$, for all $x \in X_{w_{0}}$, then for any $\epsilon>0$ there exist $C^{\prime}>0$ and a neighborhood $\omega$ of $w_{0}$ such that

$$
\left\|S_{w} x\right\|_{w} \leq C^{\prime}\left\|T_{w} x\right\|_{w}+\epsilon\|x\|_{w}
$$

for any $w \in \omega$ and all $x \in X_{w}$. Applications of this type of result to PDE's can be found in, e.g., [30].

An immediate corollary of Theorem 2.3 and Theorem 2.5 is the stability on interpolation scales of the property of being an isomorphism. Hence, for further reference we note the following.

Theorem 2.7. Assume that $\left\{T_{w}\right\}_{w \in U}$ is an interpolating family of operators between two interpolation scales $\left\{X_{w}\right\}_{w \in U}$ and $\left\{Y_{w}\right\}_{w \in U}$. Then the set of points $z \in U$ at which $T_{z}: X_{z} \rightarrow Y_{z}$ is an isomorphism is open in $U$.

Our next goal is to further extend this theorem by studying the stability of Fredholm properties on interpolation scales. We debut with a preliminary result in this direction. 
Lemma 2.8. Consider $\left\{T_{w}\right\}_{w \in U}$ an interpolating family of operators between two interpolation scales $\left\{X_{w}\right\}_{w \in U}$ and $\left\{Y_{w}\right\}_{w \in U}$, and assume that $\kappa\left(T_{z}\right)$ is bounded uniformly for $z$ in some connected compact subset $K$ of $U$. Then the dimension of $\operatorname{KerT}_{z}$ is constant on $K$ (eventually this can be $+\infty$ ).

Proof. The idea of proof is to show that for each fixed $w_{0} \in K$ there exists some positive $\epsilon$ such that if $n \in \mathbf{N}$ has $\operatorname{dim} \operatorname{Ker} T_{w_{0}} \geq n$, then also $\operatorname{dim} \operatorname{Ker} T_{z} \geq n$ for any $z \in K$ in a small neighborhood of $w_{0}$. Then the conclusion follows from the usual argument.

To this end, assume that $\kappa\left(T_{z}\right), z \in K$, is bounded above by some finite, positive $\kappa$, and recall the positive constant $D_{K}$ corresponding to $K$ as in axiom (3). Also, fix $w_{0} \in K, n \in \mathbf{N}$ with $\operatorname{dim} \operatorname{Ker} T_{w_{0}} \geq n$, and then pick an $n$-dimensional subspace of $\operatorname{Ker} T_{w_{0}}$. We shall consider next an Auerbach basis of this subspace, i.e. $x_{1}, \ldots, x_{n} \in \operatorname{Ker} T_{w_{0}} \subseteq X_{w_{0}}$ so that $\left\|x_{i}\right\|_{w_{0}}=1$ and $\left\|\sum a_{i} x_{i}\right\|_{w_{0}} \geq \max \left|a_{i}\right|$. This idea has been first employed by Cao-Sagher [8] in the context of Banach spaces. The existence of Auerbach bases for real quasi-normed spaces is obtained by maximizing the determinant just as in the real normed case and will work with only the assumption that the unit ball is closed (e.g. if the quasi-norm is continuous as we assume). The only modification necessary for complex scalars is that one seeks the point where the determinant is real and has maximum absolute value. We refer to [28], p.16; note that the argument in [31], p. 299 for the complex case is apparently incorrect.

Pick $f_{i} \in \mathcal{F}$ with $f_{i}\left(w_{0}\right)=x_{i}$ and $\left\|f_{i}\right\|_{\mathcal{F}} \leq 2,1 \leq i \leq n$. Then, for each $i$, we have $U \ni z \mapsto\left(T_{z} f_{i}(z)\right) \delta\left(z, w_{0}\right)^{-1} \in \mathcal{G}$ and $\left\|\left(T .\left(f_{i}(\cdot)\right)\right) \delta\left(\cdot, w_{0}\right)^{-1}\right\|_{\mathcal{G}} \leq 2 M D_{K}$, where the constant $M$ estimates the interpolation norm of the family $\left\{T_{w}\right\}_{w}$. Thus, in particular, for any $z \in K$, we have $\left\|T_{z}\left(f_{i}(z)\right)\right\|_{z} \leq 2 M D_{K}\left|\delta\left(z, w_{0}\right)\right|$.

At this stage, fix $z \in K$ and (using the hypothesis) pick $\tilde{x}_{i} \in X_{z}$ with $\left\|\tilde{x}_{i}\right\|_{z} \leq$ $2 \kappa M C(K)\left|\delta\left(z, w_{0}\right)\right|$ and $T_{z} \tilde{x}_{i}=T_{z} f_{i}(z)$. Consequently, $\tilde{x}_{i}-f_{i}(z)$ belongs to $\operatorname{Ker} T_{z}$. If this latter space has dimension $<n$ then there exist scalars $a_{1}, \ldots, a_{n}$ with $\max \left|a_{i}\right|=1$ and $\sum a_{i} \tilde{x}_{i}=\sum a_{i} f_{i}(z)$. Note that

$$
\left\|\sum a_{i} f_{i}(z)\right\|_{z} \leq 2 \kappa M D_{K} C(n, \rho(\mathcal{F}))\left|\delta\left(z, w_{0}\right)\right|
$$

where $C(n, \rho(\mathcal{F}))$ is some constant depending exclusively on $n$ and $\rho(\mathcal{F})$ (we may take in fact $C(n, \rho(\mathcal{F})):=n^{1 / r}$ if $\mathcal{F}$ is $r$-normed). It then follows that there exists $g \in \mathcal{F}$ with

$$
g(z)=\sum a_{i} f_{i}(z) \text { and }\|g\|_{\mathcal{F}} \leq 4 \kappa M D_{K} C(n, \rho(\mathcal{F}))\left|\delta\left(z, w_{0}\right)\right| .
$$

Going further, the function $h:=\left(g-\sum a_{i} f_{i}\right) \delta(\cdot, z)^{-1}$ belongs to $\mathcal{F}$ and

$$
\|h\|_{\mathcal{F}} \leq D_{K} \rho(\mathcal{F})\left(\|g\|_{\mathcal{F}}+\left\|\sum a_{i} f_{i}\right\|_{\mathcal{F}}\right) \leq C_{1}<+\infty
$$

where $C_{1}=C_{1}(n, K, M, \rho(\mathcal{F}))$ is some positive, finite constant. In turn, this implies that

$$
\begin{aligned}
1=\max \left|a_{i}\right| & \leq\left\|\sum a_{i} x_{i}\right\|_{w_{0}}=\left\|\sum a_{i} f_{i}\left(w_{0}\right)\right\|_{w_{0}} \\
& \leq \rho(\mathcal{F})\left(\left\|g\left(w_{0}\right)\right\|_{w_{0}}+\left\|g\left(w_{0}\right)-\sum a_{i} f_{i}\left(w_{0}\right)\right\|_{w_{0}}\right) \\
& \leq \rho(\mathcal{F})\left(\|g\|_{\mathcal{F}}+\left|\delta\left(w_{0}, z\right)\right|\|h\|_{\mathcal{F}}\right) \\
& \leq C_{2}\left(\left|\delta\left(z, w_{0}\right)\right|+\left|\delta\left(w_{0}, z\right)\right|\right),
\end{aligned}
$$


where $C_{2}=C_{2}(n, K, M, \rho(\mathcal{F}))$ is some positive, finite constant, independent of $z$, $w_{0} \in K$. For $\left|\delta\left(z, w_{0}\right)\right|+\left|\delta\left(w_{0}, z\right)\right|$ small enough this leads to a contradiction, thus the lemma is proved.

The precise statement of the Fredholm stability result alluded to earlier is contained in the next theorem. Before we state it, we make one more definition. Consider $(U, \delta, Z, \mathcal{F})$ an interpolation scale and for each closed subspace $\tilde{Z}$ of $Z$ set $\mathcal{F}_{\tilde{Z}}:=\{f \in \mathcal{F} ; f(U) \subseteq \tilde{Z}\}$. Note that if all admissible functions are continuous, then $\left(U, \delta, \tilde{Z}, \mathcal{F}_{\tilde{Z}}\right)$ becomes an interpolation scale in a natural way.

An interpolation scale as above is said to have the intersection property provided there exists a closed subspace $\tilde{Z}$ of $Z$ so that $\mathcal{F}_{\tilde{Z}} \subseteq \mathcal{F}$ densely, and for each closed subspace $E$ of $\tilde{Z}$ one has $E=\bigcap_{w \in U}\left\{f(w) ; f \in \mathcal{F}_{E}\right\}$.

A couple of comments are in order here. The above definition formalizes the idea of an interpolation scale whose intersection of all intermediate spaces is fairly rich. Indeed, it is easily checked that $\tilde{Z}$, referred to in the sequel as the intersection space, has $\tilde{Z} \subseteq \bigcap_{w \in U} X_{w}$, and that in fact $\tilde{Z}$ embeds into each $X_{w}$ continuously and densely. Furthermore, the intersection property is not so difficult to check in many concrete applications and, in fact, in the case in which the interpolation scale arises by using the classical complex method for a pair of compatible Banach spaces in the usual fashion, then the intersection property is automatically satisfied.

Theorem 2.9. Let $\left\{T_{w}\right\}_{w \in U}$ be an interpolating family of operators between two interpolation scales $\left\{X_{w}\right\}_{w \in U},\left\{Y_{w}\right\}_{w \in U}$, and suppose that $\left\{Y_{w}\right\}_{w \in U}$ has the intersection property. Then the set of points $z \in U$ at which $T_{z}: X_{z} \rightarrow Y_{z}$ is a Fredholm operator is open in $U$ and the index is locally constant on this set.

Moreover, for each $w \in U$ such that $T_{w}: X_{w} \rightarrow Y_{w}$ is Fredholm there exists a neighborhood $\omega \subseteq U$ of $w$ such that $\operatorname{dim} \operatorname{Ker} T_{z} \leq \operatorname{dim} \operatorname{Ker} T_{w}$ and $\operatorname{dim} \operatorname{Coker} T_{z} \leq$ $\operatorname{dim} \operatorname{Coker} T_{w}$ for each $z \in \omega$.

Proof. To fix notation, recall we are assuming that $\left\{Y_{w}\right\}_{w \in U} \equiv(U, \delta, W, \mathcal{G})$ has the intersection property, and we let $\tilde{W}$ denote the intersection space. Also, let $Z$ and $\mathcal{F}$ be, respectively, the ambient space and the class of admissible functions for the interpolation scale $\left\{X_{w}\right\}_{w \in U}$.

We claim that if $T_{w_{0}}$ is Fredholm for some $w_{0} \in U$ then $T_{z}$ is Fredholm with the same index for $\left|\delta\left(z, w_{0}\right)\right|$ small enough. To this effect, pick a finite dimensional subspace $E$ of $\tilde{W}$ so that $E \oplus T_{w_{0}}\left(X_{w_{0}}\right)=Y_{w_{0}}$. Augmenting $Z$ to $Z \oplus E$ and $\mathcal{F}$ to $\mathcal{F} \oplus \mathcal{F}_{E}$ it is easy to check that $\left(U, \delta, Z \oplus E, \mathcal{F} \oplus \mathcal{F}_{E}\right)$ is an interpolation scale whose intermediate spaces are $\left\{X_{w} \oplus E\right\}_{w \in U}$. Moreover, introducing $T_{w}^{\prime}(x, y):=T_{w} x+y$, then $\left\{T_{w}^{\prime}\right\}_{w \in U}$ becomes an interpolating family of operators between the scales $\left\{X_{w} \oplus E\right\}_{w \in U}$ and $\left\{Y_{w}\right\}_{w \in U}$.

Since $T_{w_{0}}^{\prime}: X_{w_{0}} \oplus E \rightarrow Y_{w_{0}}$ is onto, by Lemma 2.8, the dimension of the kernel is constant in a neighborhood of $w_{0}$ where $T_{w_{0}}^{\prime}$ remains onto. This implies that $\operatorname{dim} T_{z}^{-1}(E)$ is constant in this neighborhood. Now $\operatorname{dim}\left(\operatorname{Coker} T_{z}\right)=\operatorname{dim} E-$ $\operatorname{dim} T_{z}\left(X_{z}\right) \cap E=\operatorname{dim} E-\operatorname{dim} T_{z}^{-1}(E)+\operatorname{dim}\left(\operatorname{Ker} T_{z}\right)$ so that the index is also constant on this neighborhood. Finally, note that also $\operatorname{dim}\left(\operatorname{Coker} T_{z}\right) \leq \operatorname{dim} E=$ $\operatorname{dim}\left(\right.$ Coker $\left.T_{w_{0}}\right)$, and the conclusion follows.

We conclude this section with a global stability result.

Theorem 2.10. Let $\left\{T_{w}\right\}_{w \in U}$ be an interpolating family of operators between two interpolation scales, and suppose that $U$, the space of parameters is connected and 
that $\left\{Y_{w}\right\}_{w \in U}$ has the intersection property. Also, assume that there exists a point $w \in U$ such that $T_{w}: X_{w} \rightarrow Y_{w}$ is an isomorphism.

Then, if $\eta\left(T_{z}\right)>0$ for all $z \in U$, or if $\kappa\left(T_{z}\right)<\infty$ for all $z \in U$ it follows that $T_{z}: X_{z} \rightarrow Y_{z}$ is an isomorphism for all $z \in U$.

Proof. Let $V$ be set of $z$ where $T_{z}$ is an isomorphism. By Theorem $2.7 V$ is open. We show that $V$ is also closed. Let $v \in V$ be a boundary point and assume that $\eta\left(T_{v}\right)>0$ but $T_{v}$ is not onto. Then we can as in the previous proof augment $Z$, the ambient space for the interpolation scale $\left\{X_{w}\right\}_{w \in U}$ by some fixed, one-dimensional subspace of the intersection space so that $T_{v}^{\prime}$ still has a lower bound $\eta\left(T_{v}^{\prime}\right)>0$. However, since $T_{z}^{\prime}$ cannot be one-one for $z \in V$, this will contradict Theorem 2.5.

For the other case assume $T_{v}$ onto but not one-one. Then a similar reasoning, this time based on Lemma 2.8, leads to a contradiction and this proves the result.

\section{THE COMPLEX METHOD FOR PAIRS OF QUASI-BANACH SPACES}

The setup discussed in the previous section encompasses and unifies both complex and real interpolation in a way which avoids excessive discussion of technicalities.

Real interpolation, i.e. $(\theta, p)$-methods, can be obtained by starting from two quasi-Banach spaces $X_{0}, X_{1}$, continuously embedded in some larger topological vector space, and such that $X_{0} \cap X_{1}$ is dense in $X_{j}, j=0,1$. We take $Z:=X_{0}+X_{1}$ to be the ambient space, the annulus $U:=\{z \in \mathbf{C} ; 1<|z|<e\}$ as the space of parameters, and $\delta(z, w):=z-w$. Also, the class $\mathcal{F}$ of admissible functions consists of analytic functions of the form $f(z)=\sum_{n \in \mathbf{Z}} x_{n} z^{n}$, where $x_{n} \in X_{0} \cap X_{1}, n \in \mathbf{Z}$, and the series converges in the interior of the annulus. This latter space is equipped with the quasi-norm given by $\|f\|_{\mathcal{F}}:=\max \left\{\left(\sum_{n \in \mathbf{Z}} e^{n}\left\|x_{n}\right\|_{X_{1}}^{p}\right)^{1 / p},\left(\sum_{n \in \mathbf{Z}}\left\|x_{n}\right\|_{X_{0}}^{p}\right)^{1 / p}\right\}$. Finally, the intermediate spaces $\left\{X_{\theta}\right\}_{0<\theta<1}$ are as in (2.2) when $w=e^{\theta}, 0<\theta<1$. For more details we refer to the forthcoming paper [12].

Next we present the complex method of interpolation for pairs of quasi-Banach spaces. Let us first recall that if $Y$ is a topological vector space and $U$ is an open subset of the complex plane then a map $f: U \rightarrow Y$ is called analytic if given $z_{0} \in U$ there exists $\eta>0$ so that there is a power series expansion $f(z)=\sum_{n=0}^{\infty} y_{n} z^{n}$ converging uniformly for $\left|z-z_{0}\right|<\eta$. The theory of analytic functions with values in quasi-Banach spaces was developed in [33], [24], [25]. It is important to note here that in general there is no analogue of the Maximum Modulus Principle, but there is an important subclass of quasi-Banach spaces called A-convex in [25] in which the Maximum Modulus Principle does hold.

A quasi-Banach space $X$ is called $A$-convex if there is a constant $C$ so that for every polynomial $P: \mathbf{C} \rightarrow X$ we have $\|P(0)\|_{X} \leq C \max _{|z|=1}\|P(z)\|_{X}$. It is shown in [25] that if $X$ is A-convex it has an equivalent quasi-norm which is plurisubharmonic (i.e. we can insist that $C=1$ ). Let us also point out that being A-convex also implies that $\max _{|z| \leq 1}\|f(z)\|_{X} \leq C \max _{|z|=1}\|f(z)\|_{X}$ for any analytic function $f:\{z ;|z|<1\} \rightarrow X$ which is continuous on $\{z ;|z| \leq 1\}$.

Next, we recall some basic properties of analytic functions:

Proposition 3.1. Suppose $0<p \leq 1$ and that $m \in \mathbf{N}$ is such that $m>\frac{1}{p}$. Then there is a constant $C=C(m, p)$ so that if $X$ is a p-normed quasi-Banach space and $f: \bar{\Delta} \rightarrow X$ is a continuous function which is analytic on the unit disk 


$$
\begin{gathered}
\Delta:=\{z ;|z|<1\} \text { then for } z \in \Delta \text { we have } f(z)=\sum_{n=0}^{\infty} \frac{f^{(n)}(0)}{n !} z^{n} \text {, and } \\
\qquad\left\|f^{(n)}(0)\right\|_{X} \leq C(m+n) ! \sup _{z \in \Delta}\|f(z)\|_{X} .
\end{gathered}
$$

This is Theorem 6.1 of [24].

Proposition 3.2. Let $X$ be a quasi-Banach space and let $U$ be an open subset of the complex plane. Let $f_{n}: U \rightarrow X$ be a sequence of analytic functions. If $\lim _{n \rightarrow \infty} f_{n}(z)=f(z)$ uniformly on compacta then $f$ is also analytic.

This follows from Theorem 6.3 of [24].

Our next theorem shows that, many times in practice, the ambient space $Z$ plays only a minor role in the setup. More specifically, assume that $Z$ is a space of distributions in which a quasi-Banch space $X$ is continuously embedded. Then, having a $X$-valued function analytic for the quasinorm topology is basically the same as requiring analyticity for the weak topology (induced on $X$ from $Z$ ).

Theorem 3.3. Suppose $X$ is a quasi-Banach space and that $U$ is an open subset of the complex plane. Let $f: U \rightarrow X$ be a locally bounded function. Suppose there is a weaker Hausdorff vector topology $\tau_{0}$ on $X$ which is locally $p$-convex for some $0<p<1$ and such that $f: U \rightarrow\left(X, \tau_{0}\right)$ is analytic. Then $f: U \rightarrow X$ is analytic.

Proof. We may suppose that $p$ is such that $X$ is also $p$-normable. Consider the largest locally $p$-convex vector topology $\tau$ on $X$ which is weaker than the given quasi-norm topology and so that $f: U \rightarrow(X, \tau)$ is analytic. Next consider the quasi-normed topology $\tau^{*}$ on $X$ generated by the unit ball which is the $\tau$-closure of the given unit ball $B_{X}$ of $X$. It is easy to see that $\tau^{*}$ is given by the quasinorm $\|x\|_{*}=\sup _{j \in J}\|x\|_{j}$ where $\left\{\|\cdot\|_{j}: j \in J\right\}$ is the family of $\tau$-continuous $p$-seminorms on $X$ satisfying $\|x\|_{j} \leq\|x\|_{X}$.

We now show that $f: U \rightarrow\left(X, \tau^{*}\right)$ is analytic. To this end suppose $z_{0} \in$ $U$. Choose $r>0$ so that $\left\{z:\left|z-z_{0}\right| \leq r\right\} \subset U$ and $\sup _{\left|z-z_{0}\right| \leq r}\|f(z)\|_{X}=$ $M<\infty$. Then for each $i$ we can use Proposition 3.1 to estimate $\left\|f^{(n)}\left(z_{0}\right)\right\|_{j} \leq$ $C(m+n) ! r^{-n} M$ for suitable $m>\frac{1}{p}$ and $C=C(m, p)$. But then $\left\|f^{(n)}\left(z_{0}\right)\right\|_{*} \leq$ $C(m+n) ! r^{-n} M^{n}$ and it follows that the Taylor series $\sum_{n=0}^{\infty} \frac{f^{(n)}\left(z_{0}\right)}{n !}\left(z-z_{0}\right)^{n}$ converges in $\left(X, \tau^{*}\right)$ necessarily to $f(z)$ for $\left|z-z_{0}\right|<r$. This proves our assertion.

Now we must have $\tau=\tau^{*}$ by the choice of $\tau$. This means exactly that the identity map $i: X \rightarrow(X, \tau)$ is open (see [26] Theorem 1.4) by a form of the Open Mapping Theorem. Hence $\tau$ is the quasi-norm topology on $X$ and the result is proved.

We now let $\left(X_{0}, X_{1}\right)$ be an interpolation couple of quasi-Banach spaces, i.e. $X_{j}$, $j=0,1$, are continuously embedded into a larger topological vector space $Y$, and $X_{0} \cap X_{1}$ is dense in $X_{j}, j=0,1$. Also, let $U$ stand in this case for the strip $\{z \in \mathbf{C} ; 0<\operatorname{Re} z<1\}$, and take $Z:=X_{0}+X_{1}, \delta(z, w):=z-w$.

We then define $\mathcal{F}$, the class of admissible functions as the space of bounded, analytic functions $f: U \rightarrow X_{0}+X_{1}$ which extend continuously to the closure of the strip such that the traces $t \mapsto f(j+i t)$ are bounded continuous functions into $X_{j}, j=0,1$.

As in [22], we endow $\mathcal{F}$ with the quasi-norm

$$
\|f\|_{\mathcal{F}}:=\max \left\{\sup _{t}\|f(i t)\|_{X_{0}}, \sup _{t}\|f(1+i t)\|_{X_{1}}, \sup _{z \in U}\|f(z)\|_{X_{0}+X_{1}}\right\} .
$$


With this definition, the axioms (1)-(3) stated in section 2 are readily verified, and we denote by $X_{w}=\left[X_{0}, X_{1}\right]_{w}, w \in U$, the corresponding intermediate spaces. Of course $X_{w}$ depends only on $\Re w$ and so we typically consider $X_{\theta}$ for $0<\theta<1$. Note that the second term in the definition of $\|\cdot\|_{\mathcal{F}}$ is precisely designed to ensure both the completeness of $\mathcal{F}$ and the continuity of the evaluation functions. However, it should be pointed out that this term can be dropped as being dominated by the first if $X_{0}+X_{1}$ is A-convex.

Note that if $T$ is a linear bounded operator mapping $X_{j}$ into itself, $j=0,1$. Then $T$ is clearly bounded on $X_{0}+X_{1}$ and is therefore an interpolating operator. Interpolating analytic families of operators $\left\{T_{w}\right\}_{0<\Re w<1}$ can also be defined as in the previous section.

Our definition which is the natural extension of Calderón's original definition for Banach spaces is not, however, usually employed in the existing literature. In order to avoid discussing analytic functions in quasi-Banach spaces, authors have typically employed two variants.

One variant of this approach is to replace $\mathcal{F}$ by its closed subspace $\mathcal{F}_{0}$ generated by functions with only finite-dimensional range, and this is used in many places in the literature e.g. [22],[21] and [13]. While it is not clear in general that this leads to the same interpolation spaces, in the case we wish to consider (Hardy spaces) this makes no essential difference. The advantage here is simply that one can use functions with finite-dimensional range, without discussing the meaning of analyticity.

A second variant is to require that the functions $f \in \mathcal{F}$ be analytic into the ambient space $Z$, which is typically a locally convex space of distributions (cf. [22], $[6]$ ). Theorem 3.3 shows that this does not change $\mathcal{F}$; however if one requires only $Z$-continuity at the boundary this may result in a different space. In [2] Bernal takes $Z$ to be A-convex for certain applications.

We next point out that the method described above gives the result predicted by the Calderón formula for nice pairs of function spaces. The theorem below is due to Gomez and Milman [21] for a $\mathcal{F}_{0}$-variation of the definition. We will give the details for the convenience of the reader.

Let $(\Omega, \mu)$ be a $\sigma$-finite measure space and let $L_{0}$ be the collection of all complexvalued, $\mu$-measurable functions on $\Omega$. Recall that a quasi-Banach function space $X$ on $(\Omega, \mu)$ is an order-ideal in the space $L_{0}$ containing a strictly positive function, equipped with a quasi-norm $\|\cdot\|_{X}$ so that $\left(X,\|\cdot\|_{X}\right)$ is complete, and if $f \in X$ and $g \in L_{0}$ with $|g| \leq|f|$ a.e. then $g \in X$ with $\|g\|_{X} \leq\|f\|_{X}$.

Theorem 3.4. Let $\Omega$ be a Polish space and let $\mu$ be a $\sigma$-finite Borel measure on $\Omega$. Let $X_{0}, X_{1}$ be a pair of quasi-Banach function spaces on $(\Omega, \mu)$. Suppose that both $X_{0}$ and $X_{1}$ are $A$-convex and separable. Then $X_{0}+X_{1}$ is $A$-convex and $X_{\theta}=\left[X_{0}, X_{1}\right]_{\theta}=X_{0}^{1-\theta} X_{1}^{\theta}$, in the sense of equivalence of quasi-norms.

Remarks. $X_{0}^{1-\theta} X_{1}^{\theta}$ is the function space defined by the quasi-norm

$$
\|h\|:=\inf \left\{\|f\|_{X_{0}}^{1-\theta}\|g\|_{X_{1}}^{\theta} ;|h| \leq|f|^{1-\theta}|g|^{\theta}, f \in X_{0}, g \in X_{1}\right\} .
$$

The hypothesis of separability in this case is equivalent to order-continuity, which can be reformulated as the following property: if $g \in X_{j},(j=0,1)$ and $\left|f_{n}\right| \leq|g|$ for all $n$ and $f_{n} \rightarrow f$ a.e., then $\left\|f_{n}-f\right\|_{X_{j}} \rightarrow 0$.

Proof. We indicate briefly the argument that $X_{0}+X_{1}$ is A-convex. We may assume ([25]) that $X_{0}$ and $X_{1}$ are both $p$-convex lattices i.e. we have for $f_{1}, \ldots, f_{n} \in X_{j}$ 
we have

$$
\left\|\left(\sum_{k=1}^{n}\left|f_{k}\right|^{p}\right)^{1 / p}\right\|_{X_{j}} \leq\left(\sum_{k=1}^{n}\left\|f_{k}\right\|_{X_{j}}^{p}\right)^{1 / p}
$$

for $j=0,1$. This implies that the spaces $X_{j}^{p}:=\left\{f \in L_{0} ;|f|^{1 / p} \in X_{j}\right\}$ when normed by $\|f\|_{X_{j}^{p}}=\left\||f|^{1 / p}\right\|_{X_{j}}^{p}$ are Banach function spaces. Now suppose $f_{k} \in X_{0}+X_{1}=$ $W$ for $k=1,2, \ldots, n$. Pick $g_{k} \in X_{0}$ and $h_{k} \in X_{1}$ with $\left\|g_{k}\right\|_{X_{0}}+\left\|h_{k}\right\|_{X_{1}} \leq 2\left\|f_{k}\right\|_{W}$. Then

$$
\begin{aligned}
\left\|\left(\sum_{k=1}^{n}\left|f_{k}\right|^{p}\right)^{1 / p}\right\|_{W} & \leq\left\|\left(\sum_{k=1}^{n}\left(\left|g_{k}\right|+\left|h_{k}\right|\right)^{p}\right)^{1 / p}\right\|_{W} \\
& \leq 2^{1 / p-1}\left\|\left(\sum_{k=1}^{n}\left|g_{k}\right|^{p}\right)^{1 / p}+\left(\sum_{k=1}^{n}\left|h_{k}\right|^{p}\right)^{1 / p}\right\|_{W} \\
& \leq 2^{1 / p-1}\left\|\left(\sum_{k=1}^{n}\left|g_{k}\right|^{p}\right)^{1 / p}\right\|_{X_{0}}+2^{1 / p-1}\left\|\left(\sum_{k=1}^{n}\left|h_{k}\right|^{p}\right)^{1 / p}\right\|_{X_{1}} \\
& \leq 2^{1 / p-1}\left(\sum_{k=1}^{n}\left\|g_{k}\right\|_{X_{0}}^{p}\right)^{1 / p}+2^{1 / p-1}\left(\sum_{k=1}^{n}\left\|h_{k}\right\|_{X_{1}}^{p}\right)^{1 / p} \\
& \leq 2^{1 / p+1}\left(\sum_{k=1}^{n}\left\|f_{k}\right\|_{W}^{p}\right)^{1 / p}
\end{aligned}
$$

so that $X_{0}+X_{1}$ is also a $p$-convex lattice and so is A-convex. As in [21] we remark that the remainder of the argument is very similar to the original argument of A. P. Calderón ([5]).

First suppose $f_{0} \in X_{0}$ and $f_{1} \in X_{1}$ and suppose that $f_{0}$ and $f_{1}$ have the same supports. Then the function $z \rightarrow\left|f_{0}\right|^{1-z}\left|f_{1}\right|^{z}$ is easily shown to be in $\mathcal{F}$ (here we use the separability hypotheses on $X_{0}$ and $X_{1}$, and it follows that

$$
\left\|\left|f_{0}\right|^{1-\theta}\left|f_{1}\right|^{\theta}\right\|_{\theta} \leq C\left\|f_{0}\right\|_{X_{0}}^{1-\theta}\left\|f_{1}\right\|_{X_{1}}^{\theta}
$$

for a suitable constant $C$. This implies that for any $f \in\left[X_{0}, X_{1}\right]_{\theta}$ we have $\|f\|_{\theta} \leq$ $C\|f\|_{X_{0}^{1-\theta} X_{1}^{1-\theta}}$. (Here $C=1$ if we drop the third term in our definition of $\mathcal{F}$.)

Now suppose $F \in \mathcal{F}$. For any $q \leq p$ let $W_{q}=\left(X_{0}+X_{1}\right)^{q}$ which is also a Banach function space. We first observe that if $z_{0} \in U$ then for some $r>0$ we have a series expansion $F(z)=\sum_{n=0}^{\infty} f_{n}\left(z-z_{0}\right)^{n}$ with $f_{n} \in X_{0}+X_{1}$ valid for $\left|z-z_{0}\right|<r$ and such that $\limsup \left\|f_{n}\right\|_{X_{0}+X_{1}}^{1 / n} \leq r^{-1}$. Then $\sum_{n=0}^{\infty}\left|f_{n} \| z-z_{0}\right|^{n}$ is convergent in $X_{0}+X_{1}$ and it follows easily that we can argue pointwise to deduce that

$$
\left|F\left(z_{0}\right)(\omega)\right|^{q} \leq \frac{1}{2 \pi} \int_{0}^{2 \pi}\left|F\left(z_{0}+\rho e^{i t}\right)(\omega)\right|^{q} d t
$$

for $\mu$-a.e. $\omega \in \Omega$ and any $\rho<r$. Now $z \mapsto|F(z)|^{q}$ is continuous into $W_{q}$ and a simple application of Fubini's theorem shows that for any positive $\phi \in W_{q}^{*}$ we have $z \mapsto \phi\left(|F(z)|^{q}\right)$ is subharmonic on $U$. Thus

$$
\phi\left(|F(\theta)|^{q}\right) \leq \int_{-\infty}^{\infty} P_{0}(\theta, t) \phi\left(|F(i t)|^{q}\right) d t+\int_{-\infty}^{\infty} P_{1}(\theta, t) \phi\left(|F(1+i t)|^{q}\right) d t
$$


where $P_{0}$ and $P_{1}$ are the components of the Poisson kernel for the strip. Here $\int P_{0} d t=1-\theta$ and $\int P_{1} d t=\theta$. Let $g=\left((1-\theta)^{-1} \int_{-\infty}^{\infty} P_{0}(\theta, t)|F(i t)|^{p} d t\right)^{1 / p}$. By $p$-convexity (or by the local convexity of $W_{p}$ ) we have $g \in X_{0}$ and $\|g\|_{X_{0}} \leq\|F\|_{\mathcal{F}}$; similarly if $h=\left(\theta^{-1} \int_{-\infty}^{\infty} P_{1}(\theta, t)|F(1+i t)|^{p} d t\right)^{1 / p}$ then $\|h\|_{X_{1}} \leq\|F\|_{\mathcal{F}}$. Now if $q \leq p$ we deduce $|F(\theta)|^{q} \leq(1-\theta) g^{q}+\theta h^{q}$. Taking $\log$ of both sides and then letting $q \rightarrow 0$, this implies $|F(\theta)| \leq g^{1-\theta} h^{\theta}$ or $\|F(\theta)\|_{X_{0}^{1-\theta} X_{1}^{\theta}} \leq\|F\|_{\mathcal{F}}$.

For $0<p<\infty$ let $H^{p}=H^{p}\left(\mathbf{R}^{m}\right)$ denote the real Hardy spaces as introduced in [18]. That the family of Hardy spaces $\left\{H^{p}\left(\mathbf{R}^{m}\right)\right\}_{0<p<\infty}$ is an interpolation scale of A-convex spaces for the complex method is essentially well known (cf., e.g., [6], [13], [19], [1]), although the precise definition of complex interpolation can vary.

For our purposes, it is useful to note that a simple way to see that $\left[H^{p}, H^{q}\right]_{\theta}=H^{r}$ where $\frac{1}{r}=\frac{1-\theta}{p}+\frac{\theta}{q}$ would be to use the identification with a pair of lattices given by taking a common unconditional basis in the spaces $H_{p}, H_{q}$. This can be achieved by using a wavelet basis (cf. Theorem 7.20 of [20]) and reduces the problem to interpolating two sequence spaces $\dot{f}_{p}^{0,2}$ and $\dot{f}_{q}^{0,2}$. Now Theorem 3.4 (with $\Omega=\mathbf{N}$ and $\mu$ counting measure) can be applied; we need only the appropriate Calderón formula for the $f$-spaces and this is done in [19]. A rather similar approach is given via tent spaces in [1].

\section{Applichtions to PDE's}

Let $\Omega$ be a Lipschitz domain in $\mathbf{R}^{m}$. In the unbounded case this means that $\Omega$ is the domain above the graph of a Lipschitz function $\varphi: \mathbf{R}^{m-1} \rightarrow \mathbf{R}$, i.e. $\Omega=\left\{(\varphi(x)+t, x) ; x \in \mathbf{R}^{m-1}, t>0\right\}$. Also, in the bounded case, $\Omega$ is a bounded domain whose boundary is locally given by graphs of Lipschitz functions. Let $n(P)$ be the outward unit normal defined at almost every boundary point $P \in \partial \Omega$ and denote by $d \sigma$ the surface measure on $\partial \Omega$. Set $\sigma_{m}$ for the area of the unit sphere $S^{m-1} \subseteq \mathbf{R}^{m}$ and $\langle\cdot, \cdot\rangle$ for the usual inner product of vectors in $\mathbf{R}^{m}$ (the corresponding Euclidean norm in $\mathbf{R}^{m}$ is denoted by $\left.|\cdot|\right)$. We denote by $L^{p}(\partial \Omega)$ the Lebesgue spaces of scalar-valed functions which are $d \sigma$-measurable and $p$-th power integrable on $\partial \Omega$.

Next, recall that the classical double layer potential operator on $\partial \Omega$ acts on boudary densities $f: \partial \Omega \rightarrow \mathbf{C}$ by

$$
\mathcal{D} f(X):=\frac{1}{\sigma_{m}} \int_{\partial \Omega} \frac{\langle n(Q), Q-X\rangle}{|X-Q|^{m}} f(Q) d \sigma(Q), \quad X \in \Omega .
$$

Then, at almost every boundary point $P \in \partial \Omega$ we have the usual jump relations

$$
\begin{aligned}
\lim _{\substack{X \rightarrow P \\
X \in \gamma(P)}} \mathcal{D} f(X) & =\frac{1}{2} f(P)+\text { p.v. } \frac{1}{\sigma_{m}} \int_{\partial \Omega} \frac{\langle n(Q), Q-P\rangle}{|P-Q|^{m}} f(Q) d \sigma(Q) \\
& =:\left(\frac{1}{2} I+\mathcal{K}\right) f(P) .
\end{aligned}
$$

Here, $\gamma(P):=\{X \in \Omega ;|X-P| \leq 2 \operatorname{dist}(X, \partial \Omega)\}$ is the nontangential approach region corresponding to $P \in \partial \Omega$. Also, $I$ is the identity operator and the principal value singular integral $\mathcal{K} f$ is the so-called singular double layer potential operator acting on $f$. 
We shall also be interested to work with $\mathcal{K}^{*}$, the formal adjoint of the singular double layer potential operator, defined by

$$
\mathcal{K}^{*} f(P)=\text { p.v. } \frac{1}{\sigma_{m}} \int_{\partial \Omega} \frac{\langle n(P), P-Q\rangle}{|P-Q|^{m}} f(Q) d \sigma(Q), \quad P \in \partial \Omega .
$$

More material on these can be found in e.g. [17], [23], [35], [14].

For each $0<\alpha<1$, denote by $C^{\alpha}(\partial \Omega)$ the class of Hölder continuous functions of order $\alpha$ on $\partial \Omega$, i.e. $f \in C^{\alpha}(\partial \Omega)$ if $|f(P)-f(Q)| \leq M|P-Q|^{\alpha}$ for some finite constant $M$, uniformly for $P, Q \in \partial \Omega$. Set $\|f\|_{C^{\alpha}(\partial \Omega)}$ for the best constant in the previous inequality.

Our first result in this section is the integral representation formula contained in the following theorem.

Theorem 4.1. There exist a small, positive $\alpha_{0}$ which depends only on the dimension $m$ and the Lipschitz character of the domain $\Omega$ for which the following property holds. If a function $u$ harmonic in $\Omega$ (in the unbounded domain case $u$ is also assumed to be suitably small at infinity) assumes continuous boundary values and $\left.u\right|_{\partial \Omega} \in C^{\alpha}(\partial \Omega)$ with $0<\alpha<\alpha_{0}$, then $u$ is the double layer extension in $\Omega$ of a boundary density from the same class. In other words, $u$ can be represented in the form

$$
u(X)=\frac{1}{\sigma_{m}} \int_{\partial \Omega} \frac{\langle n(Q), Q-X\rangle}{|X-Q|^{m}} f(Q) d \sigma(Q), \quad X \in \Omega,
$$

for some boundary density $f$ which is Hölder continuous of order $\alpha$ on $\partial \Omega$. Moreover, $f$ is uniquely determined and in fact $f=\left(\frac{1}{2} I+\mathcal{K}\right)^{-1}\left(\left.u\right|_{\partial \Omega}\right)$.

Note that an immediate corollary is the classical estimate

$$
\|u\|_{C^{\alpha}(\bar{\Omega})} \leq C\|u\|_{C^{\alpha}(\partial \Omega)}
$$

valid for any function $u$ harmonic in $\Omega$, provided $\alpha>0$ is sufficiently small, depending on $\partial \Omega$. Before proceeding any further, let us also point out that similar results are valid for solutions to the Dirichlet problem with Hölder continuous boundary data for the Helmholtz operator $\triangle+k^{2}$ in Lipschitz domains. Of course, here the wave number $k \in \mathbf{C}, \operatorname{Im} k \geq 0$, is assumed not to be a Dirichlet eigenvalue for the domain under discussion.

Recall that for $\frac{m-1}{m}<p \leq 1, p$-atoms are defined as scalar-valued functions $a$ supported in a surface ball $S(P, r):=B(P, r) \cap \partial \Omega$ (for some $P \in \partial \Omega$ and some $r>$ $0)$, such that $\|a\|_{L^{\infty}} \leq \sigma(S)^{-\frac{1}{p}}$ and $\int_{\partial \Omega} a d \sigma=0$. Set $\alpha:=\frac{1-p}{p}(m-1) \in(0,1)$ so that the pairing $\int_{\partial \Omega} a h d \sigma$ between a $p$-atom $a$ and a function $h$ Hölder continuous of order $\alpha$ on $\partial \Omega$ is well defined. Then the atomic Hardy space $H_{\mathrm{at}}^{p}(\partial \Omega)$ (cf. [10]) is defined as the collection of all continuous functionals $f$ on $C^{\alpha}(\partial \Omega)$ which can be represented in the form $f=\sum_{j} \lambda_{j} a_{j}$, where $a_{j}$ 's are $p$-atoms and $\lambda_{j} \in \mathbf{R}$ are so that $\sum_{j}\left|\lambda_{j}\right|^{p}<+\infty$. It is well known that this space is complete when endowed with the natural quasi-norm

$$
\|f\|_{H_{\mathrm{at}}^{p}(\partial \Omega)}:=\inf \left\{\left(\sum_{j}\left|\lambda_{j}\right|^{p}\right)^{\frac{1}{p}} ; f=\sum_{j} \lambda_{j} a_{j}, \lambda_{j} \in \mathbf{R}, a_{j} p-\text { atom }\right\}
$$


(in fact, the above formula defines a norm if and only if $p=1$ ). Accordingly, the well-definiteness of the pairing mention above translates into

$$
\left(H_{\mathrm{at}}^{p}(\partial \Omega)\right)^{*}=C^{\alpha}(\partial \Omega) .
$$

Next, we introduce the scale $\left\{H^{p}(\partial \Omega)\right\}_{\frac{m-1}{m}<p<\infty}$ by

$$
H^{p}(\partial \Omega):=\left\{\begin{array}{l}
L^{p}(\partial \Omega), \text { if } 1<p<+\infty \\
H_{\mathrm{at}}^{p}(\partial \Omega), \text { if } \frac{m-1}{m}<p \leq 1 .
\end{array}\right.
$$

A simple observation which is of importance for us is that the $\left\{H^{p}\right\}_{p}$ scale is essentially invariant under bi-Lipschitz changes of coordinates. More concretely, if $D$ is another Lipschitz domain in $\mathbf{R}^{m}$ and $\Lambda: \partial D \rightarrow \partial \Omega$ is a bi-Lipschitz homeomorphism then, for each $\frac{m-1}{m}<p<+\infty$,

$$
H_{\mathrm{at}}^{p}(\partial \Omega) \ni f \mapsto(f \circ \Lambda)\left|J_{\Lambda}\right| \in H_{\mathrm{at}}^{p}(\partial D)
$$

is an isomorphism of quasi-Banach spaces. Here $J_{\Lambda}$ is the Jacobian of $\Lambda$ (note that $\left|J_{\Lambda}\right|$ is bounded away from zero and infinity uniformly on $\partial D$ ) and the idea is that $p$-atoms are mapped into a certain fixed multiple of $p$-atoms. This observation can be used to transfer problems formulated entirely on the scale $\left\{H^{p}(\partial \Omega)\right\}_{p}$ to more convenient ones, like $\left\{H^{p}\left(\mathbf{R}^{m-1}\right)\right\}_{p}$ (in the case of unbounded Lipschitz domains) and $\left\{H^{p}\left(S^{m-1}\right)\right\}_{p}$ (in the case of bounded Lipschitz domains).

The proof of Theorem 4.1 will be accomplished in a series of lemmas which we state below. Fix some Lipschitz domain $\Omega$ in $\mathbf{R}^{m}$. First, recall the double layer potential operator $\mathcal{D}$ from (4.1) and denote by $C^{\alpha}(\bar{\Omega})$ the space of all functions in $\Omega$ which extend as Hölder continuous functions of order $\alpha$ in $\bar{\Omega}$ endowed with the usual homogeneous norm $\|u\|_{C^{\alpha}(\bar{\Omega})}:=\sup \left\{\frac{|u(X)-u(Y)|}{|X-Y|^{\alpha}} ; X \neq Y, X, Y \in \Omega\right\}$.

Lemma 4.2. For each $0<\alpha<1, \mathcal{D}: C^{\alpha}(\partial \Omega) \rightarrow C^{\alpha}(\bar{\Omega})$ is well defined, linear and bounded.

This is perhaps well known but, since we lack an exact reference, for the sake of completeness we indicate a simple proof.

Proof. It is not too difficult to check that a continuously differentiable function $F: \Omega \rightarrow \mathbf{R}$ extends in $C^{\alpha}(\bar{\Omega})$ provided

$$
\sup _{X \in \Omega} \operatorname{dist}(X, \partial \Omega)^{1-\alpha}|\nabla F(X)| \leq C_{0}<+\infty,
$$

and that in fact $\|F\|_{C^{\alpha}(\bar{\Omega})} \leq C_{1} C_{0}$, where $C_{1}$ depends only on $\partial \Omega, m$ and $\alpha$. Next, pick $f \in C^{\alpha}(\partial \Omega), X \in \Omega$ and set $F:=\mathcal{D} f, h:=|X-P|$, where $P \in \partial \Omega$ is such that $|X-P|=\operatorname{dist}(X, \partial \Omega)$. Note that, since $\mathcal{D} 1=1$, there is no loss of generality in assuming that $f(P)=0$. Split the domain of integration in $\mathcal{D} f$ over $\{Q \in \partial \Omega ;|P-Q| \leq 100 h\}$ and $\{Q \in \partial \Omega ;|P-Q| \geq 100 h\}$. If we now bound the kernel of $\nabla \mathcal{D}$ by $C h^{m}$ in the first resulting integral and by $C|P-Q|^{m}$ in the second one, then straightforward size estimates yield (4.4).

Lemma 4.3. For any $\frac{m-1}{m}<p<+\infty$, the operator $\mathcal{K}^{*}$ is a well defined, bounded linear mapping of $H^{p}(\partial \Omega)$ into itself.

Proof. This is essentially well known too. The case $1<p<+\infty$ is basically the theorem of Coifman, McIntosh and Meyer (eventually combined with the method of rotation of Calderón and Zygmund); see e.g. [9], [34] for details. In turn, this 
can be used to show that $\mathcal{K}^{*}$ maps atoms into molecules so that case $\frac{m-1}{m}<p \leq 1$ is covered by the rather general theory in [10].

Lemma 4.4. The operator $\frac{1}{2} I+\mathcal{K}^{*}$ is an isomorphism of $H^{1}(\partial \Omega)$ onto itself.

Proof. This is Theorem 3.9 in [14].

We are now in a position to present the proof of Theorem 4.1.

Proof of Theorem 4.1. From the discussion in the last part of section 3 we know that $\left\{H^{p}\right\}_{\frac{m-1}{m}<p<\infty}$ is an interpolation scale and that $\frac{1}{2} I+\mathcal{K}^{*}$ is an interpolation operator on it. At this point, Lemma 4.3, Lemma 4.4, and the discussion in section 3 together with the results in section 2 give us that there exists a small, positive $\epsilon$ such that $\frac{1}{2} I+\mathcal{K}^{*}$ is an isomorphism of $H^{p}$ onto itself for each $1-\epsilon<p \leq 1$. Now, by duality, there exists some small positive $\alpha_{0}$ such that $\frac{1}{2} I+\mathcal{K}$ is an isomorphism of $C^{\alpha}$ onto itself for each $0<\alpha<\alpha_{0}$ (as pointed out in the introduction, this has been first observed in [4] with a completely different proof). With this at hand, the conclusion in Theorem 4.1 easily follows from Lemma 4.2 and the jump relation $(4.2)$.

Remark. It is well known that $\left\{C^{\alpha}(\partial \Omega)\right\}_{0<\alpha<1}$ is a complex interpolation scale. It then follows from the global stability result in section 2 (i.e. Theorem 2.10) that, with regard to the invertibility of $\frac{1}{2} I+\mathcal{K}$ on $C^{\alpha}(\partial \Omega)$, what breaks down for $\alpha$ close to 1 (on general Lipschitz domains) is precisely the closedness of the range of this operator.

The same considerations apply, for instance, to the operator $\frac{1}{2} I-\mathcal{K}^{*}$ when acting on $L_{0}^{p}(\partial \Omega)$ for $p$ large $(p>2)$.

Recall next the single layer potential operator $\mathcal{S}$ which acts on boundary densities by

$$
\mathcal{S} f(P):=\int_{\partial \Omega} \frac{1}{|P-Q|^{m-1}} f(Q) d \sigma(Q),
$$

and let $\nabla_{\tan }:=\nabla-n \frac{\partial}{\partial n}$ be the usual tangential gradient operator on $\partial \Omega$. Following [14], we say that $a: \partial \Omega \rightarrow \mathbf{R}$ is a regular atom if $\operatorname{supp} a \subseteq S(P, r)$ for some $P \in \partial \Omega$, $r>0$ and $\left\|\nabla_{\tan } a\right\|_{L^{\infty}(\partial \Omega)} \leq \sigma(S)^{-\frac{1}{p}}$. Then define $H_{\mathrm{at}}^{p, 1}(\partial \Omega)$ as the $l^{p}$-span of regular atoms.

Theorem 4.5. Let $\Omega$ be a Lipschitz domain in $\mathbf{R}^{m}$. Then there exists a small, positive $\epsilon=\epsilon(\partial \Omega, m)$ such that the operator $\mathcal{S}: H_{\mathrm{at}}^{p}(\partial \Omega) \rightarrow H_{\mathrm{at}}^{p, 1}(\partial \Omega) / \mathbf{R}$ is an isomorphism for each $1-\epsilon<p \leq 1$.

Proof. The case $p=1$ is contained in [14] and, hence, the conclusion follows by the same pattern by proving that $H_{\mathrm{at}}^{p, 1}(\partial \Omega) / \mathbf{R}$ is an interpolation scale for the complex method. In fact, this last point can be circumvented as follows. First, using the results in [14] and Theorem 2.5 we see that there exists $\epsilon>0$ such that

$$
\|f\|_{H_{\mathrm{at}}^{p}(\partial \Omega)} \leq C\left\|\nabla_{\tan } S f\right\|_{H_{\mathrm{at}}^{p}\left(\partial \Omega, \mathbf{R}^{3}\right)} \leq C\|S f\|_{H_{\mathrm{at}}^{p, 1}\left(\partial \Omega, \mathbf{R}^{3}\right)}
$$

for any $f \in H_{\mathrm{at}}^{p}(\partial \Omega), 1-\epsilon<p \leq 1$. In particular, $\mathcal{S}: H_{\mathrm{at}}^{p}(\partial \Omega) \rightarrow H_{\mathrm{at}}^{p, 1}(\partial \Omega) / \mathbf{R}$ is injective with closed range. Now the conclusion follows from the fact that $\mathcal{S}$ : $L_{0}^{2}(\partial \Omega) \rightarrow L^{2,1}(\partial \Omega) / \mathbf{R}$ is an isomorphism; see [34]. 
Our last application concerns the Lamé system of linear elastostatics on a Lipschitz domain $\Omega$ in $\mathbf{R}^{3}$

$$
\mu \triangle \vec{u}+(\lambda+\mu) \nabla(\operatorname{div} \vec{u})=\overrightarrow{0} \text { in } \Omega
$$

where $\mu>0$ and $\lambda>-\frac{2}{3} \mu$ are the so-called Lamé constants. Natural boundary conditions for $(4.5)$ can be obtained by prescribing

$$
\left.\vec{u}\right|_{\partial \Omega}=\vec{g}
$$

(i.e. Dirichlet boundary conditions), or

$$
\frac{\partial \vec{u}}{\partial \nu}:=\left.\left(\lambda(\operatorname{div} \vec{u}) n+\mu\left[\nabla \vec{u}+\nabla \vec{u}^{t}\right] n\right)\right|_{\partial \Omega}=\vec{h},
$$

where the boundary conormal derivative operator $\partial / \partial \nu$ is called traction or stress. Note that the superscript $t$ indicates transpose of the $3 \times 3$ matrix $\nabla \vec{u}=\left(\partial_{i} u_{j}\right)_{i, j}$.

Recall the Kelvin matrix $\Gamma=\left(\Gamma_{i, j}\right)_{i, j}$ of fundamental solutions for the system of elastostatics, where for $1 \leq i, j \leq 3$

$$
\Gamma_{i, j}(X):=\frac{1}{2 \omega_{2}}\left(\frac{1}{\mu}+\frac{1}{2 \mu+\lambda}\right) \frac{\delta_{i, j}}{|X|}+\frac{1}{2 \omega_{2}}\left(\frac{1}{\mu}-\frac{1}{2 \mu+\lambda}\right) \frac{X_{i} X_{j}}{|X|^{3}} .
$$

See [27]. For vector valued densities on $\partial \Omega$ we define the single and the double layer potential operators by

$$
\mathbf{S} \vec{f}(X):=\int_{\partial \Omega} \Gamma(X-Q) \vec{f}(Q) d \sigma(Q), \quad X \in \mathbf{R}^{3},
$$

and by

$$
\mathbf{D} \vec{f}(X):=\int_{\partial \Omega}\left(\frac{\partial}{\partial \nu(Q)} \Gamma(X-Q)\right)^{t} \vec{f}(Q) d \sigma(Q), \quad X \in \Omega,
$$

respectively. Here the operator $\partial / \partial \nu$ applies to each column of the matrix $\Gamma$.

Finally, we record the corresponding jump relation

$$
\begin{aligned}
\lim _{\substack{X \in \gamma(P) \\
X \in \gamma}} \mathbf{D} \vec{f}(X) & =\frac{1}{2} \vec{f}(P)+\text { p.v. } \int_{\partial \Omega}\left(\frac{\partial}{\partial \nu(Q)} \Gamma(X-Q)\right)^{t} \vec{f}(Q) d \sigma(Q) \\
& =:\left(\frac{1}{2} \mathbf{I}+\mathbf{K}\right) \vec{f}(P), \quad P \in \partial \Omega,
\end{aligned}
$$

and let $\mathbf{K}^{*}$ denote the formal adjoint of $\mathbf{K}$. See [16] for more details.

Theorem 4.6. Let $\Omega$ be a Lipschitz domain in $\mathbf{R}^{3}$. Then there exists a small, positive $\epsilon$ such that $\frac{1}{2} \mathbf{I}+\mathbf{K}^{*}$ is an isomorphism of $H_{a t}^{p}\left(\partial \Omega, \mathbf{R}^{3}\right)$ onto itself for each $1-\epsilon<p \leq 1$.

Also, there exists some small positive $\alpha_{0}$ such that $\frac{1}{2} \mathbf{I}+\mathbf{K}$ is an isomorphism of $C^{\alpha}\left(\partial \Omega, \mathbf{R}^{3}\right)$ onto itself for each $0<\alpha<\alpha_{0}$.

Proof. The fact that $\mathbf{K}^{*}$ maps $H_{\text {at }}^{p}\left(\partial \Omega, \mathbf{R}^{3}\right)$ boundedly into itself for each $\frac{m-1}{m}<$ $p \leq 1$ follows essentially as before (cf. Lemma 4.3). Also, by combining Lemma 1.6 in [15] with the techniques in [14] one can show that $\frac{1}{2} \mathbf{I}+\mathbf{K}^{*}$ is an isomorphism of $H_{\text {at }}^{1}\left(\partial \Omega, \mathbf{R}^{3}\right)$. Now the first part of the theorem follows from this, the discussion in section 3 and the stability results in section 2 . The second part is a direct corollary of this and (4.3). 
The next theorem provides an alternative approach to results of B. Dahlberg and C. Kenig (see [15] for a proof based on different techniques). Let us point out that the layer potential integral representation formula (4.9) is new.

Theorem 4.7. Let $\Omega$ be a Lipschitz domain in $\mathbf{R}^{3}$. Then there exists some small positive $\alpha_{0}$ such that for each $0<\alpha<\alpha_{0}$ the boundary value problem (4.5)-(4.6) has a unique solution $\vec{u} \in C^{\alpha}\left(\bar{\Omega}, \mathbf{R}^{3}\right)$ for any boundary data $\vec{g} \in C^{\alpha}\left(\partial \Omega, \mathbf{R}^{3}\right)$.

Moreover, we have the integral representation formula

$$
\vec{u}=\mathbf{D} \vec{f} \quad \text { in } \Omega
$$

with $\vec{f}:=\left(\frac{1}{2} \mathbf{I}+\mathbf{K}\right)^{-1} \vec{g} \in C^{\alpha}\left(\partial \Omega, \mathbf{R}^{3}\right)$, and the natural estimate $\|\vec{u}\|_{C^{\alpha}\left(\bar{\Omega}, \mathbf{R}^{3}\right)} \leq$ $C\|\vec{g}\|_{C^{\alpha}\left(\partial \Omega, \mathbf{R}^{3}\right)}$ holds for some $C=C(\alpha, \partial \Omega)>0$.

Proof. This is a direct corollary of Theorem 4.6 and the jump relation (4.8) much in the spirit of the proof of Theorem 4.1 (note that the obvious analogue of Lemma 4.2 remains valid for the case we are considering here).

Further consequences of the above theorems in the study of elliptic boundary value problems in non-smooth domains are analyzed in [29]. We conclude with a number of observations of independent interest.

Remarks. (1) Approximating continuous functions by Hölder continuous on the boundary and relying on $L^{\infty}$ estimate from [15], it follows from Theorem 4.7 that solutions $\vec{u}$ of (4.5)-(4.6) with $\vec{g}$ continuous are in fact continuous up to and including the boundary of the domain.

(2) Let us note that Theorem 4.6 is also important for proving regularity results for the Green function associated with the Lamé system.

(3) Theorem 4.6 should have important applications in the numerical treatment of (4.5)-(4.6). For instance, it may be used to establish error estimates for the so called collocation method.

(4) Another remark of interest is that for Lipschitz domains with a more complicated underlying topology most boundary integral operators are only Fredholm. In this case, Theorem 2.9 can be utilized. See [30].

(5) Finally, we want to stress that our techniques point to somewhat similar results for the heat operator $\partial_{t}-\triangle$ in Lipschitz cylinders as well. The corresponding $H^{1}$-atomic theory for the adjoint of the caloric double layer potential operator has been worked out in [3].

\section{REFERENCES}

1. J. Alvarez and M. Milman, Interpolation of tent spaces and applications, in "Function Spaces and Applications", M. Cwikel et al. eds., Springer LN, No. 1302, 1988, 11-21. MR 89i:46025

2. A. Bernal, Some results on complex interpolation of $T_{q}^{p}$-spaces, Israel Math. Conf. Proc. $\mathbf{5}$ (1992), 1-10. MR 94b:46101

3. R. Brown, The initial-Neumann problem for the heat equation in Lipschitz cylinders, Trans. Amer. Math. Soc. 170 (1990), 1-52. MR 90k:35112

4. R. Brown, The Neumann problem on Lipschitz domains in Hardy spaces of order less than one, Pacific J. of Math. 171 (1995), 389-408. MR 97m:35048

5. A. P. Calderón, Intermediate spaces and interpolation, the complex method, Studia Math. 24 (1964), 113-190. MR 29:5097

6. A. P. Calderón and A. Torchinsky, Parabolic maximal functions associated with a distribution, II, Adv. in Math. 24 (1977), 101-171. MR 56:9180 
7. W. Cao and Y. Sagher, Stability of Fredholm properties on interpolation scales, Ark. för Math. 28 (1990), 249-258. MR 92e:46146

8. W. Cao and Y. Sagher, Stability in interpolation of families of Banach spaces, Proc. of Amer. Math. Soc. 112 (1991), 91-100. MR 91h:46123

9. R. Coifman, A. McIntosh and Y. Meyer, L'intégrale de Cauchy définit un opérateur borné sur $L^{2}$ pour les courbes Lipschitziennes, Ann. of Math. 116 (1982), 361-387. MR 84m:42027

10. R. Coifman and G. Weiss, Extensions of Hardy spaces and their use in analysis, Bull. Amer. Math. Soc. 83 (1977), 569-645. MR 56:6264

11. R. R. Coifman, M. Cwikel, R. Rochberg, Y. Sagher and G. Weiss, A theory of complex interpolation for families of Banach spaces, Advances in Math. 43 (1982), 203-229. MR 83j: 46084

12. M. Cwikel, N. J. Kalton, M. Milman and R. Rochberg, in preparation.

13. M. Cwikel, M. Milman and Y. Sagher, Complex interpolation of some quasi-Banach spaces, J. Funct. Anal. 65 (1986), 339-347. MR 87h:46152

14. B. E. J. Dahlberg and C. E. Kenig, Hardy spaces and the $L^{p}-$ Neumann problem for Laplace's equation in a Lipschitz domain, Ann. of Math. 125 (1987), 437-465. MR 88d:35044

15. B. E. J. Dahlberg and C. E. Kenig, $L^{p}$-estimates for the three dimensional system of elastostatics on Lipschitz domains, in Lecture Notes in Pure and Appl. Math., Vol. 122, Cora Sadosky ed., 1990, 621-634. MR 91h:35053

16. B. E. J. Dahlberg, C. E. Kenig and G. C. Verchota, Boundary value problems for the system of elastostatics on Lipschitz domains, Duke Math. J. 57 (1988), 795-818. MR 90d:35259

17. E. Fabes, Layer potential methods for boundary value problems on Lipschitz domains, in Potential Theory, Surveys and Problems, J. Král et al. eds., Lecture Notes in Math., No. 1344, Springer-Verlag, 1988, 55-80. MR 89m:31001

18. C. Fefferman and E. M. Stein, $H^{p}$ spaces of several variables, Acta Math. 129 (1972), 137-193. MR 56:6263

19. M. Frazier and B. Jawerth, A discrete transform and decompositions of distribution spaces, J. Funct. Anal. 93 (1990), 34-171. MR 92a:46042

20. M. Frazier, B. Jawerth and G. Weiss, Littlewood-Paley theory and the study of function spaces, CBMS Regional Conference Series 79, Amer. Math. Soc., Providence, 1989. MR 92m:42021

21. M. Gomez and M. Milman, Complex interpolation of $H^{p}$-spaces on product domains, Ann. Math. Pura Appl. 155 (1989), 103-115. MR 91g:46030

22. J. Janson and P. W. Jones, Interpolation between $H^{p}$ spaces: The complex method, J. Funct. Anal. 48 (1982), 58-80. MR 84c:46021

23. D. S. Jerison and C. E. Kenig, Boundary value problems on Lipschitz domains, in "Studies in Partial Differential Equations", Studies in Math., M.A.A. 23, W. Littman ed. (1982), 1-68. MR 85f:35057

24. N. J. Kalton, Analytic functions in non-locally convex spaces, Studia Math. 83 (1986), 275303. MR 88a: 46022

25. N. J. Kalton, Plurisubharmonic functions on quasi-Banach spaces, Studia Math 84 (1986), 297-324. MR 88g:46030

26. N. J. Kalton, N. T. Peck and J. W. Roberts, An F-space sampler, London Math. Soc., Lecture Notes Series, No. 89, 1984. MR 87c:46002

27. O. A. Ladyzhenskaya, The mathematical theory of viscous incompressible flow, Gordon and Breach, 1963. MR 40:7610

28. J. Lindenstrauss and L. Tzafriri, Classical Banach spaces II, Function spaces, Springer, Berlin, 1979. MR 81c:46001

29. E. Fabes, O. Mendez and M. Mitrea, Boundary layers on Sobolev-Besov spaces and Poisson's equation for the Laplacian domain, to appear in J. Funct. Anal. (1998).

30. D. I. Mitrea, M. Mitrea, and M. Taylor, preprint (1996).

31. S. Rolewicz, Metric linear spaces, D. Reidel, Dordrecht, 1984. MR 88i:46004b

32. I. Y. Šneiberg, Spectral properties of linear operators in interpolation families of Banach spaces, Mat. Issled. 9 (1974), 214-229. MR 58:30362

33. P. Turpin, Convexités dans les espaces vectoriels topologiques generaux, Dissertationes Math. 131 (1974). MR 54:11028

34. G. Verchota, Layer potentials and regularity for the Dirichlet problem for Laplace's equation in Lipschitz domains, J. Funct. Anal. 59 (1984), 572-611. MR 86e:35038 
35. A. T. Vignati and M. Vignati, Spectral theory and complex interpolation, J. Funct. Anal. 80 (1988), 387-397. MR 90a:47011

36. S. Zaremba, Sur le principe de Dirichlet, Acta Math. 34 (1911), 293-316.

Department of Mathematics, University of Missouri-Columbia, Columbia, Missouri 65211

E-mail address: nigel@math.missouri.edu

Department of Mathematics, University of Missouri-Columbia, Columbia, Missouri 65211

E-mail address: marius@math.missouri.edu

The Institute of Mathematics of the Romanian Academy, P.O. Box 1-764, RO-70700 Bucharest, Romania 